DOI: 10.12957/demetra.2016.18029

\title{
Percepção materna do estado nutricional de crianças em escolas municipais de Porto Alegre-RS
}

\author{
Maternal perception of child nutritional status from public schools in Porto Alegre-RS, Brazil
}

\author{
Roberta Roggia Friedrich' \\ Thiago Antunes? \\ Ilaine Schuch ${ }^{3}$ \\ 1 Universidade Federal do Rio Grande do \\ Sul, Faculdade de Medicina, Programa de \\ Pós Graduação em Saúde da Criança e do \\ Adolescente. Porto Alegre-RS, Brasil. \\ ${ }^{2}$ Universidade Federal do Rio Grande do Sul, \\ Faculdade de Medicina, Departamento de \\ Nutrição. Porto Alegre-RS, Brasil. \\ ${ }^{3}$ Universidade Federal do Rio Grande do Sul, \\ Faculdade de Medicina, Departamento de \\ Nutrição, Centro de Estudos em Alimentação e \\ Nutrição Porto Alegre-RS, Brasil. \\ Financiamento: Este estudo foi financiado pela \\ Pró-reitoria de Extensão da Universidade Federal \\ do Rio Grande do Sul através do edital PROEXT \\ 2013. Roberta Roggia Friedrich foi financiada \\ como bolsista de doutorado pelo Conselho \\ Nacional de Desenvolvimento Científico e \\ Tecnológico (CNPq). \\ Correspondência / Correspondence \\ Roberta Roggia Friedrich \\ E-mail: robertafriedrich@hotmail.com
}

\section{Resumo}

Objetivo: Avaliar a percepção materna do estado nutricional de crianças de escolas municipais de ensino fundamental de Porto Alegre-RS. Metodologia: Estudo transversal, realizado no início do primeiro semestre letivo de 2013, com 495 escolares de ambos os sexos, matriculados do primeiro ao quarto ano, do ensino fundamental de 12 escolas municipais da cidade de Porto Alegre-RS, e suas respectivas mães. A concordância entre o estado nutricional das crianças e a percepção materna sobre o mesmo foi avaliada através do teste de Kappa ponderado. $\mathrm{O}$ teste qui-quadrado foi utilizado para verificar a associação entre a percepção materna do estado nutricional da criança por sexo, estado nutricional da mãe e estado nutricional da criança. As análises foram consideradas significativas quando o valor de $\mathrm{P}$ encontrado era $\leq 0,05$. Resultados: Quanto ao estado nutricional, $0,2 \%$ das crianças apresentaram baixo-peso; $61,0 \%$, eutrofia e $38,4 \%$, excesso de peso. Uma parcela de $51,6 \%$ das crianças com excesso de peso teve seu estado nutricional subestimado por suas mães (Kappa $=0,36 ; \mathrm{P}<0,001)$, sendo $59,1 \%$ entre os meninos e 44,3\% entre as meninas (Kappa $=0,27 ; \mathrm{P}<0,001)$. Conclusão: A percepção materna distorcida do estado nutricional do filho pode prejudicar a adoção de estilo de vida mais saudável e resultar no desenvolvimento de doenças crônicas. Estratégias de saúde pública focadas no entendimento do excesso de peso como um problema de saúde e na importância do ambiente familiar são o primeiro passo no esforço para prevenir a obesidade.

Palavras-chave: Obesidade Infantil; Percepção; Imagem Corporal; Estado Nutricional; Saúde Escolar; Distorção da Percepção. 


\section{Abstract}

Objective: To evaluate maternal perception of child nutritional status of elementary school from public schools in Porto Alegre, Rio Grande do Sul state, Brazil. Methods: Cross-sectional study realized at the beginning of the first semester of 2013 with 495 schoolchildren of both sexes, enrolled of first to fourth grade of elementary school in 12 schools in Porto Alegre, with their respective mother. The agreement between the nutritional status of children and maternal perception has been assessed through the weighted Kappa test. The chi-square test was used to assess the association between maternal perception of child nutritional status by sex, maternal nutritional status and child nutritional status. The results were considered significant when $P$ value was $\leq 0,05$. Results: About nutritional status, $0.2 \%$ of children were underweight, $61.0 \%$ were eutrophic and $38.4 \%$ overweight. About $51.6 \%$ of children with overweight had their nutritional status underestimated by their mothers (Kappa $=0.36$; $\mathrm{P}<0.001$ ), being $59.1 \%$ among boys and $44.3 \%$ among girls with overweight (Kappa $=0.27 ; \mathrm{P}<0.001)$. Conclusion: The distorted maternal perception of the nutritional status of the child may affect the adoption of healthier lifestyles and result in development of chronic diseases. Public health strategies focused on understanding the severity of overweight as a health problem and the importance of the family environment are the first step in the campaign to prevent obesity.

Key words: Pediatric Obesity; Perception; Body Image; Nutritional Status; School Health; Perceptual Distortion.

\section{Introdução}

A obesidade tem sido uma preocupação mundial, inclusive no Brasil. Os dados mais atuais e de abrangência nacional sobre o estado nutricional da população infantil brasileira são os da Pesquisa de Orçamentos Familiares (POF) de 2008 a 2009 e da Pesquisa Nacional sobre Demografia e Saúde (PNDS) de 2006, para crianças até cinco anos.

O resultado da POF mostrou que a prevalência de excesso de peso variou de $32 \%$ a $40 \%$ em crianças de cinco a nove anos no Sudeste, Sul e Centro-Oeste e de 25\% a 30\% no Norte e Nordeste, faixa etária em que o aumento da prevalência da obesidade foi mais intenso e a PNDS registrou 
prevalência nacional de sobrepeso de $6,6 \% .^{2}$ No âmbito escolar, a prevalência de excesso de peso foi de $23 \%$ entre alunos do nono ano do ensino fundamental de escolas brasileiras, de acordo com a Pesquisa Nacional dos Escolares (PENSE). ${ }^{3}$

A compreensão dos pais de que a obesidade é um problema de saúde deve ser o primeiro passo na promoção de um estilo de vida saudável, resultando em um peso corporal mais saudável entre as crianças. ${ }^{4}$ Os pais que percebem o excesso de peso de seus filhos são mais propensos a fazer mudanças de seus estilos de vida, influenciando, desta forma, a saúde dos filhos. ${ }^{5}$ A adequada percepção materna em relação ao estado nutricional do seu filho pode resultar na busca por profissionais de saúde, o que se torna de grande importância para o processo de prevenção, diagnóstico e tratamento da obesidade na infância. ${ }^{6-9}$

É necessário ressaltar que a intervenção na base familiar, sobretudo com o envolvimento dos pais na promoção de hábitos saudáveis, deve ser contemplada e estimulada durante o tratamento da obesidade, uma vez que crianças são influenciadas pelos hábitos de seus pais. ${ }^{10}$ Por isso, as orientações introduzidas pelos profissionais de saúde devem ser seguidas em casa por meio de exemplos positivos dos pais para filhos, com alimentação saudável e prática regular de exercício físico. Além disso, programas de intervenções apresentam melhores resultados no controle da obesidade quando as estratégias utilizadas incluem o componente familiar.,11,

Com o atual aumento da prevalência de obesidade infantil no mundo, é evidente a necessidades de ações que busquem a prevenção e o tratamento da mesma. Portanto, é fundamental a percepção dos familiares sobre o estado nutricional de seus filhos.

Para acrescentar informações na literatura, o presente estudo busca avaliar a percepção materna do estado nutricional de crianças de escolas municipais de ensino fundamental de Porto Alegre-RS.

\section{Metodologia}

Trata-se de estudo transversal com utilização de dados da avaliação da etapa-base da pesquisa intitulada "Efeito de um programa de intervenção com educação nutricional e atividade física na prevenção da obesidade em escolares: um estudo controlado randomizado". São crianças em idade escolar matriculadas do primeiro ao quarto ano, do ensino fundamental das escolas municipais da cidade de Porto Alegre-RS, e suas mães.

A cidade de Porto Alegre-RS tem a maior concentração urbana da Região Sul e a quinta mais populosa do Brasil. Abriga atualmente mais de 1,4 milhão de habitantes e seu Índice de Desenvolvimento Humano (IDH) é de 0,805, classificado como muito alto. 
Foram considerados critério de inclusão: escolares de ambos os sexos, que estavam regularmente matriculados entre o primeiro e o quarto ano, do turno da tarde, do ensino fundamental, das escolas municipais da cidade de Porto Alegre-RS, cujo responsável pelo preenchimento do questionário de pesquisa era a mãe. Foram excluídos do estudo os alunos cujo respondente do questionário não era a mãe e aquelas crianças com alguma incapacidade de realizar as medidas antropométricas, além dos alunos que não concordaram em realizar as medidas antropométricas (peso e altura), apesar do consentimento materno.

O estudo-base para o cálculo do tamanho da amostra inicial foi a metanálise apresentada por Friedrich et al., ${ }^{13}$ que avaliaram o efeito das intervenções com educação nutricional e atividade física sobre o índice de massa corporal em crianças e adolescentes, no âmbito escolar. O cálculo do tamanho da amostra foi realizado no Programa Power and Sample Size. Para um nível de significância de $5 \%$ e um poder estatístico de $90 \%$, para detectar uma diferença padronizada das médias de 0,45 sobre o índice de massa corporal, o tamanho da amostra calculado foi de 105 indivíduos para cada grupo, considerando perdas de $15 \%$, e o efeito do desenho por conglomerado $(2,0)$ o tamanho total da amostra foi aumentado para 250 em cada grupo, totalizando 500 indivíduos. No entanto, depois de finalizada a coleta de dados antropométricos, o número de escolares avaliados totalizou 600 indivíduos, sendo reduzido para o número final de 495 escolares após agrupar os questionários cujo respondente foi a mãe. Para compor a amostra do estudo, foram incluídas 12 escolas municipais, sendo que apenas três turmas de cada escola foram selecionadas aleatoriamente a participar do estudo, totalizando 1.041 alunos.

Os dados do estudo foram coletados no início do ano letivo de 2013 por uma equipe de estudantes do Curso de Nutrição da Universidade Federal do Rio Grande do Sul (UFRGS). A equipe foi treinada pelo Laboratório de Avaliação Nutricional da UFRGS para a realização de medidas antropométricas padronizadas e cuidados na utilização e calibração dos equipamentos.

A coleta de dados ocorreu em duas etapas, sendo que na primeira foram coletados, em duplicata, os dados antropométricos dos estudantes como peso $(\mathrm{kg})$ e altura $(\mathrm{cm})$. A segunda etapa consistiu na entrega de um questionário autoaplicável ao responsável, no qual constavam questões referentes à percepção materna sobre o estado nutricional do escolar.

A aferição do peso e da altura seguiu as recomendações da Organização Mundial da Saúde (OMS). ${ }^{14} \mathrm{O}$ peso foi aferido com as crianças descalças e com o mínimo de roupa possível, posicionadas no centro da plataforma da balança, com os pés juntos e braços estendidos ao longo do corpo. A altura foi medida com a criança descalça, na posição ereta, braços estendidos ao longo do corpo, calcanhares, panturrilhas, glúteos, escápula e parte posterior da cabeça encostados no estadiômetro vertical, e a cabeça posicionada no plano de Frankfurt. 
Foi adotado o valor médio das duas medidas para o cálculo do índice de massa corporal (IMC), definido como a relação entre o peso em quilogramas e a altura em metros elevada ao quadrado $\left(\mathrm{kg} / \mathrm{m}^{2}\right)$. Para a classificação do estado nutricional, utilizou-se o IMC, com pontos de corte baseados no escore $\mathrm{Z}$ para idade e sexo, preconizado pelo Ministério da Saúde, que adota os critérios utilizados pela OMS, ${ }^{15,16}$ utilizando o programa AnthroPlus para crianças.

A percepção materna do estado nutricional do escolar foi avaliada pelo instrumento de escala verbal composto da seguinte questão fechada, que era feita aos pais: "Você considera que o seu filho para a idade e altura está com?”, com três opções de resposta: baixo-peso, peso normal ou acima do peso. Além disso, o peso e a altura da mãe foram autorrelatados.

Os dados foram digitados no programa EpiData versão 3.1 em dupla digitação, a fim de verificar consistência das informações. As variáveis contínuas foram descritas através da média e desvio-padrão. Já as variáveis categóricas foram descritas através de frequência absoluta e relativa.

Para verificar concordância entre o estado nutricional das crianças e a percepção materna sobre o mesmo foi utilizado o teste de KAPPA ponderado. O teste qui-quadrado foi utilizado para verificar a associação entre a percepção materna do estado nutricional da criança por sexo, estado nutricional da mãe e estado nutricional da criança.

As análises foram consideradas significativas quando o valor de $\mathrm{P}$ encontrado era $\leq 0,05$. Os dados foram analisados pelo programa Statistical Package for Social Sciences (SPSS) versão 18.0.

O grande estudo foi cadastrado na Plataforma Brasil e aprovado pelo Comitê de Ética do Hospital de Clínicas de Porto Alegre (HCPA/UFRGS). Certificado de Apresentação para Apreciação Ética (CAE): 12406713.6.0000.5327 e número do parecer: 215.661 e registrado no Registro de Ensaios Clínicos (REBEC) número RBR- 2xx2z4.

\section{Resultados}

O presente estudo foi composto por uma amostra de 495 escolares com média de idade de 7,8 anos, havendo uma pequena predominância do sexo feminino (53,7\%), cuja distribuição mostrouse homogênea. As características da amostra estão descritas na tabela 1.

Quanto ao estado nutricional, a maioria delas encontrava-se com o peso dentro das faixas de normalidade, mas a proporção de crianças diagnosticadas com excesso de peso foi de 38,4\%, conforme apresentado na tabela 1 . 
Tabela 1. Características gerais da amostra de escolares da cidade de Porto Alegre-RS, 2013.

\begin{tabular}{lc}
\hline \multicolumn{1}{c}{ Variáveis } & $\mathrm{N}=(495)$ \\
\hline Idade (anos) Média (DP) & $7,8(1,4)$ \\
Sexo, n (\%) & $229(46,3)$ \\
Masculino & $266(53,7)$ \\
Feminino & \\
Escolaridade, n (\%) & $115(23,2)$ \\
$1^{\text {o }}$ ano & $116(23,4)$ \\
$2^{\text {o }}$ ano & $143(28,9)$ \\
3o ano & $121(24,4)$ \\
$4^{\text {o }}$ ano & \\
Antropometria & $31,08(9,7)$ \\
Média (DP) do Peso, $\mathrm{Kg}$ & $129,60(9,6)$ \\
Média (DP) da Altura, $\mathrm{cm}$ & \\
Estado Nutricional & $102(20,6)$ \\
Baixo-peso & $1(0,2)$ \\
Eutrófico & $304(61,4)$ \\
Sobrepeso & $88(17,8)$ \\
Obesidade & \\
\hline
\end{tabular}

Em relação à percepção materna do estado nutricional, 51,6\% das crianças com excesso de peso tiveram seu estado nutricional subestimado por suas mães. Entretanto, $2 \%$ das crianças que eram consideradas eutróficas tiveram seu estado nutricional superestimado por sua mãe, que as considerou com excesso de peso. Além disso, observou-se concordância, embora fraca, entre a percepção materna e o estado nutricional de seu filho determinado na avaliação antropométrica $($ Kappa $=0,36 \mathrm{P}<0,001)($ tabela 2). 
Tabela 2. Distribuição dos escolares segundo o estado nutricional e a percepção materna. Porto Alegre-RS, 2013.

\begin{tabular}{lccc}
\hline & \multicolumn{3}{c}{ Estado Nutricional } \\
& Baixo-peso & Peso normal & Excesso de peso \\
\cline { 2 - 4 } Percepção materna & $\mathrm{n}(\%)$ & $\mathrm{n}(\%)$ & $\mathrm{n}(\%)$ \\
\hline Baixo-peso & 0 & $48(15,8)$ & $2(1,1)$ \\
Peso normal & $1(100)$ & $250(82,2)$ & $98(51,6)$ \\
Excesso de peso & 0 & $6(2,0)$ & $90(47,4)$ \\
Total & $1(100)$ & $304(100)$ & $190(100,0)$ \\
\hline
\end{tabular}

Kappa $=0,36 ; \mathrm{P}<0,001$

Quando estratificados por sexo da criança, os resultados acerca da percepção materna do estado nutricional mostraram que $59,1 \%$ dos meninos e 44,3\% das meninas com excesso de peso tiveram o estado nutricional subestimado pela mãe, evidenciando uma tendência de mais mães de meninos subestimarem o excesso de peso de seu filho. Também se observou que 19,3\% dos meninos com peso adequado foram classificados como baixo-peso por suas mães. Em relação à superestimação de peso, mães, tanto de meninos quanto de meninas, superestimaram o estado nutricional de seus filhos de forma similar, classificando erroneamente 2,2\% dos meninos e 1,8\% das meninas com peso adequado como tendo excesso de peso, conforme apresentado na tabela 3. 
Tabela 3. Distribuição dos escolares de acordo com o sexo e estado nutricional, segundo a percepção materna. Porto Alegre-RS, 2013.

\begin{tabular}{|c|c|c|c|c|c|c|}
\hline \multirow[b]{3}{*}{$\begin{array}{l}\text { Percepção } \\
\text { materna }\end{array}$} & \multicolumn{6}{|c|}{$\begin{array}{l}\text { Estado nutricional da criança } \\
\qquad \mathrm{N}=495\end{array}$} \\
\hline & \multicolumn{3}{|c|}{$\begin{array}{c}\text { Masculino } \\
\mathrm{n}=229\end{array}$} & \multicolumn{3}{|c|}{$\begin{array}{l}\text { Feminino } \\
\mathrm{n}=266\end{array}$} \\
\hline & $\begin{array}{c}\text { Baixo- } \\
\text { peso } \\
n(\%)\end{array}$ & $\begin{array}{c}\text { Peso } \\
\text { adequado } \\
\text { n (\%) }\end{array}$ & $\begin{array}{c}\text { Excesso de } \\
\text { peso } \\
\text { n (\%) }\end{array}$ & $\begin{array}{c}\text { Baixo- } \\
\text { peso } \\
\mathrm{n}(\%)\end{array}$ & $\begin{array}{c}\text { Peso } \\
\text { adequado } \\
\text { n (\%) }\end{array}$ & $\begin{array}{c}\text { Excesso de } \\
\text { peso } \\
\text { n }(\%)\end{array}$ \\
\hline Baixo-peso & 0 & $26(19,3)$ & 0 & 0 & $22(13)$ & $2(2,1)$ \\
\hline Peso adequado & $1(100,0)$ & $106(78,5)$ & $55(59,1)$ & 0 & $144(85,2)$ & $43(44,3)$ \\
\hline Excesso de peso & 0 & $3(2,2)$ & $38(40,9)$ & 0 & $3(1,8)$ & $52(53,6)$ \\
\hline Total & 1 & $135(100,0)$ & $93(100,0)$ & 0 & $169(100,0)$ & $97(100,0)$ \\
\hline
\end{tabular}

Kappa $=0,27 ; \mathrm{P}<0,001$

\section{Discussão}

A maior proporção das crianças avaliadas foi classificada como adequado estado nutricional $(61,4 \%)$, sendo este resultado condizente com a maioria dos estudos publicados, cujos valores encontram-se entre $55,1 \%$ e $65 \%$, assim como para o excesso de peso $(38,4 \%)$ que também se assemelha aos resultados encontrados em outros estudos, cujos valores variaram de $20 \%$ até $40 \%{ }^{17-21}$ A distorção entre a percepção materna do estado nutricional do filho avaliada neste estudo mostrase semelhante aos resultados encontrados por Boa-Sorte et al. ${ }^{6}$ que encontrou um Kappa igual a 0,434 e por Molina et al. ${ }^{22}$ que encontrou Kappa igual a 0,217, em estudos conduzidos no Brasil.

Em outro estudo, publicado por Wake et al., ${ }^{23}$ os autores concluíram que grande parte da amostra de pais não reconheceu que seus filhos estavam acima do peso ou com obesidade. E ainda, $22 \%$ classificaram como baixo-peso as crianças que apresentavam peso normal e $63 \%$ como excesso de peso, crianças com obesidade.

Flores-Peña et al. ${ }^{9}$ também encontraram valores semelhantes em relação a distorções na percepção materna, cuja avaliação se deu mediante o questionamento similar utilizado no presente estudo. Constatou-se associação entre a percepção materna inadequada do peso do filho e a 
prevalência alta de obesidade infantil, sugerindo que estratégias de saúde pública para aumentar a consciência dos pais do real estado nutricional de seus filhos constitui o primeiro passo no esforço para prevenir a obesidade na infância.

Estudo de revisão de literatura focada em artigos que avaliaram principalmente a percepção materna, identificou, em cinco dos oito estudos analisados, a percepção materna equivocada do estado nutricional de seu filho, tendendo a subestimar o peso através da imagem corporal quando a criança apresenta excesso de peso. ${ }^{24}$

A relação entre a percepção materna e o excesso de peso de seus filhos também foi examinada em outro estudo transversal, realizado na Argentina por Hirschler et al..${ }^{25} \mathrm{em}$ que foram avaliadas 321 crianças pré-escolares, com idade média de 4,39 anos, de ambos os sexos. A análise a respeito da percepção materna identificou diferença significativa na distorção da percepção da imagem corporal entre as mães de crianças com peso normal (17\%) e mães de crianças com sobrepeso ou risco de sobrepeso (87,5\%). Entre as mães de crianças com sobrepeso, 23,7\% acreditavam que seu filho realmente apresentava sobrepeso e apenas 1,6\% dessas mães consideravam que o risco para sobrepeso era real.

Giacomossi et al. (2011) ${ }^{8}$ descrevem que estados nutricionais extremos, como obesidade e desnutrição, geram distorções na percepção dos pais, enquanto deveriam ser diagnosticados com maior facilidade pelos responsáveis pelas crianças, pois os sinais clínicos se tornam mais perceptíveis visualmente. Contudo, mães de crianças com obesidade ou desnutrição apresentaram prevalência praticamente cinco vezes maior de classificar erroneamente o estado nutricional infantil, as classificando como eutróficas.

No presente estudo, a percepção materna se mostrou associada ao sexo da criança, sendo que as mães subestimaram o estado nutricional de seus filhos do sexo masculino mais do que mães de crianças do sexo feminino. Vários outros estudos também já demonstraram esta relação. ${ }^{26,27-30}$ Alguns autores sugerem que isso ocorre em função da maior atenção que as mães dão ao estado nutricional das filhas pela importância que a sociedade confere a imagem corporal das meninas. ${ }^{6,22}$

A dificuldade em reconhecer o estado nutricional das crianças pelas mães pode estar relacionada com crenças pessoais, familiares e valores culturais, sendo obstáculos para a adoção de comportamentos mais saudáveis. . $4,31,32^{2}$

Outros fatores que influenciam na percepção materna sobre o peso corporal dos filhos têm sido amplamente discutidos na literatura acerca do tema, sendo as características dos pais como peso, nível socioeconômico, escolaridade materna, idade materna, IMC materno; e características dos filhos como peso, Índice de Massa Corporal, idade e sexo se mostraram potenciais preditores da percepção. ${ }^{33}$ 
A percepção adequada do estado nutricional das crianças deve ser elemento primordial no tratamento de obesidade na infância, principalmente pelo fato de a construção dos hábitos alimentares se dar no núcleo familiar, sendo influenciado pelo ambiente e pelas atitudes da família. Assim, a adequada percepção tanto da família quanto da criança promove possivelmente a melhor adesão, e também é requisito para a procura de tratamento. ${ }^{34}$

O reconhecimento do excesso de peso das crianças possivelmente poderá promover uma melhora na adesão aos cuidados. Além disso, pode ser o requisito necessário para a procura de ajuda profissional. Contudo, as mães tendem a considerar a obesidade de seu filho como um problema individual, ao invés de perceber que é um problema multifatorial no qual o ambiente familiar exerce grande influência na geração e manutenção da obesidade. ${ }^{24} \mathrm{Com}$ isso, as famílias têm o papel fundamental no desenvolvimento e na promoção da saúde das crianças. ${ }^{9,24}$ Por esse motivo, as práticas terapêuticas devem envolver a família e apoiar o relacionamento entre pais e filhos, e não somente focar na criança ou na relação mãe-filho.

Quanto às limitações, a inclusão de um número pequeno de vaiáveis em nossa pesquisa não permitiu explorar os fatores envolvidos no erro da percepção materna do estado nutriconal do seu filho. E ainda, estudos indicam que a utilização da escala verbal pode levar a uma elevada subestimação do excesso de peso e obesidade dos filhos, quando comparada a escala visual. ${ }^{34,35}$ Por isso, outras pesquisas utilizando maior número de variáveis, incluindo a escolaridade e idade materna, além de questões culturais da mãe e da família, peso da criança ao nascer, e avaliações utilizando técnicas qualitativas poderão contribuir para esclarecer outros aspectos deste tema.

\section{Conclusão}

Os resultados mostram que a percepção materna a respeito do peso corporal do seu filho tende a ser distorcida, principalmente quando a criança apresenta excesso de peso. As mães subestimam mais o excesso de peso nos meninos, quando comparadas às meninas.

Para uma estratégia de promoção da saúde a partir da adoção de um estilo de vida mais saudável, é importante reconhecer o estado nutricional dos filhos por parte dos pais, dentro dos critérios clínicos. Para isso destaca-se como fundamental a abordagem apropriada do tema por parte dos profissionais de saúde com as famílias. Contudo, mais estudos que visem a uma melhor compreensão da relação obesidade na infância e percepção materna, e interação com os outros fatores já identificados na literatura são necessários para que a prevenção e o tratamento da obesidade sejam eficazes. 


\section{Agradecimentos}

Agradecemos a colaboração de todos os bolsistas envolvidos neste estudo; ao Programa de Pósgraduação em Saúde da Criança e do Adolescente da Universidade Federal do Rio Grande do Sul e ao Grupo de Pesquisa de Pós-graduação do Hospital de Clínicas de Porto Alegre. Agradecemos também aos trabalhadores das escolas e à Secretaria Municipal da Educação de Porto Alegre, em especial às nutricionistas, pela receptividade e apoio no desenvolvimento das atividades nas escolas.

\section{Referências}

1. Instituto Brasileiro de Geografia e Estatística. Pesquisa de orçamentos familiares 2008-2009: antropometria e estado nutricional de crianças, adolescentes e adultos no Brasil. Rio de Janeiro: IBGE; 2010.

2. Brasil. Ministério da Saúde. Pesquisa Nacional de Demografia e Saúde da Criança e da Mulher - PNDS 2006: dimensões do processo reprodutivo e da saúde da criança. Brasília: Ministério da Saúde; 2009.

3. Instituto Brasileiro de Geografia e Estatística. Pesquisa Nacional de Saúde do Escolar 2009. Rio de Janeiro: IBGE; 2009.

4. Stanton B, Cole M, Galbraith J, Li X, Pendleton S, Cottrel L, et al. Randomized trial of a parent intervention: parents can make a difference in long-term adolescent risk behaviors, perceptions, and knowledge. Arch. Pediatr. Adolesc. Med. 2004; 158(10):947-55.

5. Rhee KE, Lago CW, Arscott-Mills T, Mehta SD. Factors associated with parental readiness to make changes for overweight children. Pediatrics 2005; 116(1):e94-e101.

6. Boa-Sorte N, Neri LA, Leite MEQ, Brito SM, Meirelles AR, Luduvice FB, et al. Percepção materna e autopercepção do estado nutricional de crianças e adolescentes de escolas privadas. J. Pediatr. 2007; 83(4):349-56.

7. Chuproski P, Mello DFd. Percepção materna do estado nutricional de seus filhos. Rev. Nutr. 2009; 22(6):929-36.

8. Giacomossi MC, Zanella T, Hõfelmann DA. Percepção materna do estado nutricional de crianças de creches de cidade do Sul do Brasil. Rev. Nutr. 2011; 24(5):689-702.

9. Flores-Peña Y, Cárdenas-Villarreal VM, Trejo-Ortiz PM, Ávila-Alpirez H, Ugarte-Esquivel A, Gallegos-Martínez J. Acciones y problemas maternos para manejar el peso del hijo de acuerdo a la percepción materna del peso y edad del hijo. Nutrición Hospitalaria 2014; 29(4):822-828.

10. Arpini LSB, Barbosa GC, Justo GF, Salaroli LB, Molina MCB. Associação entre a percepção materna do estado nutricional do filho e a qualidade da dieta de escolares. DEMETRA: Alimentação, Nutrição \& Saúde 2015; 10(2):299-314.

11. Epstein LH. Family-based behavioural intervention for obese children. Int. J. Obes. Relat. Metab. Disord. 1996; 20 (Suppl 1):S14-S21. 
12. Katz DL, O’Connell M, Njike VY, Yeh MC, Nawaz H. Strategies for the prevention and control of obesity in the school setting: systematic review and meta-analysis. Int. J. Obes. (Lond). 2008; 32(12):1780-1789.

13. Friedrich RR, Schuch I, Wagner MB. Effect of interventions on the body mass index of school-age students. Rev. Saude Publica 2012; 46(3):551-60.

14. World Health Organization. Physical status: the use and interpretation of anthropometry: report of a WHO Expert Committee. Geneva: World Health Organization; 1995.

15. Onis M, Onyango AW, Borghi E, Siyam A. Development of a WHO growth reference for school-aged children and adolescents. Geneva: Bulletin of the World Health Organization; 2007.

16. World Health Organization. WHO Child growth standards: length/height-for-age, weightforage,weight-for-length, weight-for-height and body mass index-for-age: methods and development. Geneva: WHO; 2006.

17. Schommer VA, Barbiero SM, Cesa CC, Oliveira R, Silva AD, Pellanda LC. Excesso de peso, variáveis antropométricas e pressão arterial em escolares de 10 a 18 anos. Arq. Bras. Cardiol. 2014; 102(4):312-318.

18. Ribas SA, Silva LCSd. Fatores de risco cardiovascular e fatores associados em escolares do Município de Belém, Pará, Brasil. Cad. Saúde Pública 2014; 30(3):577-586.

19. Azambuja APO, Netto-Oliveira ER, Oliveira AAB, Azambuja MA, Rinaldi W. Prevalência de sobrepeso/obesidade e nível econômico de escolares. Rev. Paul. Pediatr. 2013; 31(2):166-71.

20. Rosaneli CF, Auler F, Manfrinato CB, Rosaneli CF, Sganzerla C, Bonatto MG, et al. Avaliação da prevalência e de determinantes nutricionais e sociais do excesso de peso em uma população de escolares: análise transversal em 5.037 crianças. Rev. Assoc. Med. Bras. 2012; 58(4):472-476.

21. Reuter CP, Burgos LT, Camargo MD, Possuelo LG, Reckziegel MB, Reuter EM, et al. Prevalence of obesity and cardiovascular risk among children and adolescents in the municipality of Santa Cruz do Sul, Rio Grande do Sul. São Paulo Med. J. 2013; 131(5):323-30.

22. Molina MC, Faria CP, Montero P, Cade NV. Correspondence between children's nutritional status and mothers' perceptions: a population-based study. Cad. Saúde Pública 2009; 25(10):2285-90.

23. Wake M, Nicholson JM, Hardy P, Smith K. Preschooler obesity and parenting styles of mothers and fathers: Australian National Population Study. Pediatrics 2007; 120(6):e1520-e1527.

24. Camargo APPM, Barros Filho AA, Antonio MARGM, Giglio JS. A não percepção da obesidade pode ser um obstáculo no papel das mães de cuidar de seus filhos. Ciênc. Saúde Coletiva 2013; 18(2):323-333.

25. Hirschler V, González C, Cemente G, Talgham S, Petticnichio H, Jadzinsky M. Cómo perciben las madres de niños de jardín de infantes a sus hijos con sobrepeso? Arch. Argent. Pediatr. 2006; 104(3):221-6.

26. Tenorio A, Cobayashi F. Obesidade infantil na percepção dos pais: perception of childhood obesity by parents. Rev. Paul. Pediatr. 2011; 29(4):634-9. 
27. Hudson E, McGloin A, McConnon A. Parental weight (mis) perceptions: factors influencing parents' ability to correctly categorise their child's weight status. Matern. Child Health J. 2012; 16(9):1801-1809.

28. Vuorela N, Saha MT, Salo MK. Parents underestimate their childs overweight. Acta Paediatr. 2010; 99(9):1374-1379.

29. De La O A, Jordan KC, Ortiz K, Moyer-Mileur LJ, Stoddard G, Friedrichs M, et al. Do parents accurately perceive their child's weight status? J. Pediatr. Health Care 2009; 23(4):216-221.

30. He M, Evans A. Are parents aware that their children are overweight or obese? Do they care? Can. Fam. Physician 2007; 53(9):1493-1499.

31. Aparício G, Cunha M, Duarte J, Pereira A. Olhar dos pais sobre o estado nutricional das crianças pré-escolares. Millenium 2011; 40:99-113.

32. Crawford PB, Gosliner W, Anderson C, Strode P, Becerra-Jones Y, Samuels S, et al. Counseling latina mothers of preschool children about weight issues: suggestions for a new framework. J. Am. Diet Assoc. 2004; 104(3):387-394.

33. Al-Qaoud NM, Al-Shami E, Prakash P. Kuwaiti mothers' perception of their preschool children's weight status. J. Dev. Behav. Pediatr. 2010; 31(6):505-10.

34. Lazzeri G, Casorelli A, Giallombardo D, Grasso A, Guidoni C, Menoni E, et al. Nutritional surveillance in Tuscany: maternal perception of nutritional status of 8-9 y-old school-children. J. Prev. Med. Hyg. 2006; 47(1):16-21.

35. Eckstein KC, Mikhail LM, Ariza AJ, Thomson JS, Millard SC, Binns HJ. Parents' perceptions of their child's weight and health. Pediatrics 2006; 117(3):681-90. 
